\title{
Post-ERCP Bleeding in the Era of Multiple Antiplatelet Agents
}

Hyoung-Chul Oh ${ }^{1,2}$, Ihab I. El Hajj ${ }^{1}$, Jeffrey J. Easler ${ }^{1}$, James Watkins ${ }^{1}$, Evan L. Fogel ${ }^{1}$, Lee McHenry ${ }^{1}$, Glen A. Lehman ${ }^{1}$, Jung Sik Choi ${ }^{3}$, Hyun Kang ${ }^{4}$, and Stuart Sherman ${ }^{1}$

${ }^{1}$ Division of Gastroenterology and Hepatology, Indiana University School of Medicine, Indianapolis, IN, USA, ${ }^{2}$ Division of Gastroenterology, Chung-Ang University College of Medicine, Seoul, ${ }^{3}$ Division of Gastroenterology, Inje University Busan Paik Hospital, Inje University College of Medicine, Busan, and ${ }^{4}$ Department of Anesthesiology and Pain Medicine, Chung-Ang University College of Medicine, Seoul, Korea

Background/Aims: This study aimed to determine the risk of post-endoscopic retrograde cholangiopancreatography (post-ERCP) bleeding among patients taking antiplatelet agents (APAs), particularly in the era of multiple APAs. Methods: The primary outcomes were the frequency, type, and severity of ERCP-related bleeding according to the use of APAs. Results: The frequencies of post-ERCP bleeding among the four different groups were 16 of 2,083 (0.8\%) in the no drug group, 12 of 256 (4.7\%) in the aspirin group, 3 of $48(6.3 \%)$ in the single APA group, and 4 of $48(8.3 \%)$ in the multiple APA group $(p<0.001)$. In the univariate analysis, post-ERCP bleeding was associated with age, pull-type sphincterotomy, and APA and was inversely associated with balloon dilation of the biliary orifice. In the multivariate analysis, pull-type sphincterotomy (odds ratio [OR], 7.829; 95\% confidence interval [Cl], 1.411 to $43.453 ; p=0.019$ ) and country (Korea: OR, $0.124 ; 95 \% \mathrm{Cl}, 0.042$ to $0.361 ; p<0.001$ ) were associated with post-ERCP bleeding. Conclusions: The frequency of post-ERCP bleeding was statistically higher in patients on any APA within 6 days prior to ERCP. However, in the multivariate analysis, APA use was not associated with post-ERCP bleeding. Until a large, adequately powered study to detect differences is performed, caution is recommended when considering invasive procedures during ERCP in patients on APAs. (Gut Liver 2018;12:214-218)

Key Words: Cholangiopancreatography, endoscopic retrograde; Hemorrhage; Platelet aggregation inhibitors

\section{INTRODUCTION}

Bleeding is one of the major adverse events related to therapeutic endoscopic retrograde cholangiopancreatography (ERCP). Its reported occurrence rate ranges from $0.3 \%$ to $2.0 \% .^{1-3}$ Definite risk factors for post-sphincterotomy bleeding are reported to be coagulopathy, anticoagulation medication within 3 days of sphincterotomy, cholangitis before ERCP, bleeding during initial sphincterotomy, and lower endoscopist ERCP case volume. In contrast, factors not related to an increased risk of bleeding include aspirin (acetylsalicylic acid, ASA) or nonsteroidal anti-inflammatory drug use, ampullary tumor, longer length sphincterotomy, and extension of prior sphincterotomy. ${ }^{4}$

Antithrombotic therapy, especially with antiplatelet agents (APA), is increasingly utilized for a variety of indications and newly developed agents have been released during the last few decades. The combination of multiple APAs rather than ASA alone, has become a popular clinical practice.

American Society for Gastrointestinal Endoscopy and European Society of Gastrointestinal Endoscopy guidelines recommend holding these APAs in both elective and urgent ERCP settings. ${ }^{5,6}$ However, ERCP including sphincterotomy and balloon dilation of the biliary orifice may be necessary in urgent or emergency settings. The precise risk of performing ERCP interventions in patients taking APAs within a few days or even the ERCP day has not been clearly defined. The current study aimed to determine the true occurrence rate of post-ERCP bleeding among those patients who are taking APAs, especially in the era of multiple agents, and to compare the incidence of bleeding among the following groups: no drug use, ASA alone, single APA other than ASA, and multiple APAs.

\footnotetext{
Correspondence to: Stuart Sherman

Division of Gastroenterology and Hepatology, Indiana University School of Medicine, 550 N. University Boulevard, Suite 1634, Indianapolis, IN 46202, USA

Tel: +1-317-944-0925, Fax: +1-317-968-1265, E-mail: ssherman@iu.edu

Received on May 8, 2017. Revised on July 4, 2017. Accepted on July 4, 2017. Published online December 8, 2017

pISSN 1976-2283 eISSN 2005-1212 https://doi.org/10.5009/gnl17204

(a) This is an Open Access article distributed under the terms of the Creative Commons Attribution Non-Commercial License (http://creativecommons.org/licenses/by-nc/4.0) which permits unrestricted non-commercial use, distribution, and reproduction in any medium, provided the original work is properly cited.
} 


\section{MATERIALS AND METHODS}

\section{Procedures}

The ERCP procedures were performed by nine expert endoscopists (seven from Indiana University, USA, and two from Chung-Ang University and Inje University, Korea) in practice at high volume, academic centers with comprehensive ERCP databases. The ERCP database of the three individual institutions contains information regarding all ERCP procedures performed since July 2012 (Indiana University) and January 2014 (ChungAng University and Inje University). Data were collected and entered immediately after each case. The database contains 95 categories and more than 1,000 potential variables and includes information on demographics, clinical history, procedural indications, procedural details, procedural findings, diagnoses, complications, and follow-up. Delayed adverse events were added systematically after a review of each case at 2 weeks or when we were notified from a scheduled follow-up or by an outside physician. This study was approved by three individual Institutional Review Boards (IRB number: 1606176507) with the requirement for informed consent waived.

\section{Outcomes and data collection}

The primary outcomes of interest in this analysis were the frequency, type, severity and treatment of ERCP-related bleeding according to the use of APA. ASA and other APAs were categorized based on their method of action (dipyridamole - Persantine $^{\circledR}$; cilostazol - Pletal ${ }^{\circledR}$; thienopyridine, clopidogrel - Plavix ${ }^{\circledR}$; prasugrel - Effient ${ }^{\circledR}$; ticlopidine - Ticlid $^{\circledR}$; ticagrelor - Brilinta $\left.{ }^{\circledR}\right)^{5}$ Patients who were taking anticoagulants including heparin and warfarin were excluded in this study as the anticoagulants were generally held or invasive procedures were postponed as recommended. Bleeding time or platelet function test were not performed. Patients with known bleeding disorders such as von Willebrand disease or hemophilia were excluded. Among the 12,717 ERCP cases from three institutions, only those patients who underwent their first therapeutic ERCP were identified and enrolled in this analysis. For purposes of this study, therapeutic ERCP was defined as any procedure where an intervention that could provoke bleeding was performed such as pull-type sphincterotomy, any kind of needle-knife sphincterotomy (including precut), and balloon dilation of the biliary orifice. After identifying those patients who were taking APAs within 6 days of an ERCP, medical records were reviewed to collect the following information: underlying disease, days APA held (if any) prior to ERCP, procedural details, presence and severity of postERCP bleeding, and treatment of bleeding. The doses of individual drugs were not tallied. The electrosurgical current mode used for endoscopic sphincterotomy was ENDO CUT (ERBE USA, Marietta, GA, USA) in all three institutions. Post-ERCP bleeding and its severity were defined by established consensus criteria and graded mild, moderate, or severe. ${ }^{7}$ Post-ERCP bleeding was further categorized based on timing of presentation: early bleeding was defined as that occurring during ERCP and late bleeding when occurring after the end of the ERCP procedure. ${ }^{8}$ The bleeding was defined as self-limited when the bleeding episode ceases spontaneously without any therapeutic intervention during ERCP procedure. Endoscopic treatments of ERCP-associated bleeding consist of tamponade by compression with balloon catheter, injection therapy with diluted epinephrine around the sphincterotomy site or biliary orifice, bipolar circumactive probe cautery, and argon plasma coagulation at the site of bleeding or exposed vessel. The resumption of APAs after ERCP was at the discretion of managing physicians and was not controlled or tallied in the study. It was common practice to resume APAs in three days after ERCP.

\section{Statistical analysis}

The proportions of patients with ERCP-related bleeding were compared with the chi-square tests followed by Bonferroni correction method to adjust p-value. Select characteristics were tested by univariate analysis as potential risk factors for ERCP-related bleeding using logistic regression. A multivariate analysis was performed using factors that had univariate $\mathrm{p}$ values of $<0.20$ on logistic regression as well as age and sex. The multicollinearity diagnostic indicated no multicollinearity issues (condition indices <30; variance inflation factor values $<10$ ) between the chosen independent variables in this study.

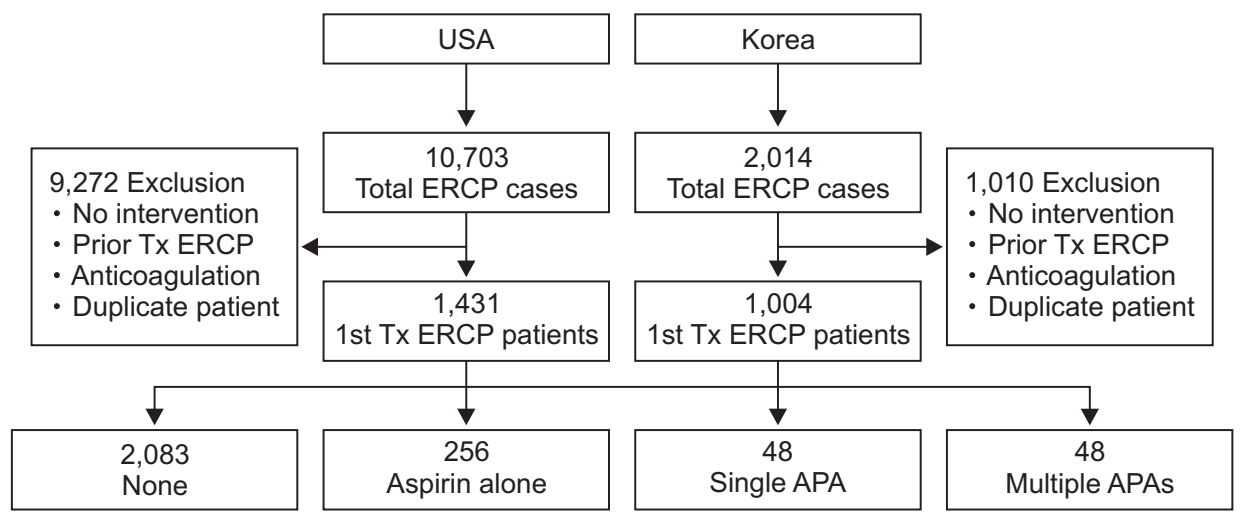

Fig. 1. Flow chart of patient enrollment.

Tx, therapeutic; ERCP, endoscopic retrograde cholangiopancreatography; APA, antiplatelet agent. 
A $p<0.05$ was considered to be significant. Results from multivariate logistic regression analysis were considered definitive because it determined variables independently associated with ERCP-related bleeding after adjusting for the contributions of the other variables. Therefore, univariate statistical tests should be taken as descriptive only because these $p$-values were not corrected for multiple testing of outcome data arising from individual patients. All analysis was performed using SPSS version 18.0 (SPSS Inc., Chicago, IL, USA).

\section{RESULTS}

\section{Patient enrollment}

One thousand and four hundred thirty-one and 1,004 patients from United States and Korea, respectively, met the inclusion criterial and were enrolled in the study. The flow diagram for patient enrollment is summarized in Fig. 1.

\section{Baseline patient characteristics}

The baseline patient characteristics including age, gender, type of drug taken, and therapeutic intervention performed, are summarized in Table 1. The enrolled patients were categorized into four groups according to the type of drugs taken: no drug $(n=2,083)$, aspirin $(n=256)$, single APA other than ASA $(n=48)$, and multiple APAs $(\mathrm{n}=48)$.

\section{Outcomes}

The frequency, severity, timing and treatment of post-ERCP bleeding are shown in Table 2. Post-ERCP bleeding occurred in 16 of 2,083 (0.8\%) in no drug group, 12 of 256 (4.7\%) in aspirin group, three of 48 (6.3\%) in single APA group, and four of 48 $(8.3 \%)$ in multiple APAs group $(\mathrm{p}<0.001)$. When compared with the no drug group, the frequencies of post-ERCP bleeding were statistically higher in the aspirin, single APA, and multiple APA group $(\mathrm{p}<0.05)$. However, there was no significant difference in

Table 1. Baseline Characteristics of the Enrolled Subjects

\begin{tabular}{|c|c|c|c|c|}
\hline Characteristic & No drug $(n=2,083)$ & Aspirin $(n=256)$ & Single APA $(n=48)$ & Multiple APA $(n=48)$ \\
\hline Age, yr & $58.0(44-71)$ & $73.0(63-79)$ & $77.0(71-81)$ & $71.0(66-79)$ \\
\hline Male sex & $891(42.8)$ & $152(59.4)$ & $26(54.2)$ & $26(54.2)$ \\
\hline \multicolumn{5}{|l|}{ Drug } \\
\hline Aspirin & 0 & 256 & 0 & 48 \\
\hline \multicolumn{5}{|l|}{ APA other than ASA } \\
\hline Dipyridamole & 0 & 0 & 4 & 1 \\
\hline Cilostazol & 0 & 0 & 3 & 4 \\
\hline Thienopyridine $(\mathrm{C} / \mathrm{P} / \mathrm{T})$ & 0 & 0 & $41(41 / 0 / 0)$ & $43(42 / 1 / 0)$ \\
\hline \multicolumn{5}{|l|}{ Intervention* } \\
\hline Pull-type sphincterotomy & 1,242 & 173 & 27 & 29 \\
\hline Needle-knife sphincterotomy & 573 & 38 & 7 & 7 \\
\hline Balloon dilation of biliary orifice & 693 & 104 & 23 & 26 \\
\hline
\end{tabular}

Data are presented as median (range), number (\%), or number.

APA, antiplatelet agent; ASA, acetylsalicylic acid; C, clopidogrel; P, prasugrel; T, ticlopidine.

*More than one intervention was performed in some patients.

Table 2. Frequency of Post-ERCP Bleeding

\begin{tabular}{|c|c|c|c|c|c|c|}
\hline & No drug $(\mathrm{n}=2,083)$ & Any APA $(n=352)$ & Aspirin (n=256) & Single APA $(n=48)$ & Multiple APA ( $\mathrm{n}=48)$ & p-value \\
\hline Bleeding & $16(0.8)$ & $19(5.4)^{*}$ & $12(4.7)^{\dagger}$ & $3(6.3)^{\dagger}$ & $4(8.3)^{\dagger}$ & $<0.001$ \\
\hline Mild/moderate/severe & $15 / 1 / 0$ & $16 / 3 / 0$ & $11 / 1 / 0$ & $1 / 2 / 0$ & $4 / 0 / 0$ & \\
\hline Early/late & $10 / 6$ & $15 / 4$ & $11 / 1$ & $1 / 2$ & $3 / 1$ & \\
\hline \multicolumn{7}{|l|}{ Treatment } \\
\hline Self-limited & 4 & 6 & 4 & 0 & 2 & \\
\hline Tamponade & 1 & 0 & 0 & 0 & 0 & \\
\hline Injection & 5 & 11 & 7 & 2 & 2 & \\
\hline Coagulation & 6 & 2 & 1 & 1 & 0 & \\
\hline
\end{tabular}

Data are presented as number (\%) or number.

ERCP, endoscopic retrograde cholangiopancreatography; APA, antiplatelet agent.

${ }^{*} \mathrm{p}<0.001$ when compared with the no drug group; ${ }^{\dagger} \mathrm{p}<0.05$ when compared with the no drug group. 
Table 3. Univariate Analysis of the Risk Factors for Post-ERCP Bleeding

\begin{tabular}{lcc}
\hline \multicolumn{1}{c}{ Factor } & OR (95\% CI) & p-value \\
\hline $\begin{array}{l}\text { Age } \\
\text { Sex }\end{array}$ & $1.023(1.001-1.045)$ & 0.037 \\
$\quad$ Male & 1 & \\
Female & $0.769(0.395-1.500)$ & 0.441 \\
Country & & \\
USA & 1 & \\
Korea & $0.489(0.228-1.048)$ & 0.066 \\
Intervention & & \\
Pull-type sphincterotomy & $11.004(2.634-45.964)$ & 0.001 \\
Needle-knife sphincterotomy & $0.268(0.082-0.877)$ & 0.029 \\
Balloon dilation of the biliary orifice & $0.119(0.049-0.292)$ & $<0.001$ \\
Drug & & \\
None & 1 & \\
ASA alone & & \\
Single APA other than ASA & $8.608(2.422-30.592)$ & 0.001 \\
Multiple APA & $12.012(3.855-37.427)$ & $<0.001$ \\
Duration of drug held prior to ERCP & & 0.807 \\
0-2 vs 3-6 & $1.132(0.418-3.066)$ & \\
\hline
\end{tabular}

ERCP, endoscopic retrograde cholangiopancreatography; OR, odds ratio; CI, confidence interval; ASA, acetylsalicylic acid; APA, antiplatelet agent.

frequencies of post-ERCP bleeding among aspirin, single APA and multiple APAs groups. In the treatment groups, post-ERCP bleeding occurred in 11 patients (58\%) with 0 drug held day, three patients $(16 \%)$ with 1 to 2 drug held days, five patients (26\%) with 3 to 6 drug held days. Most cases of post-ERCP bleeding were mild (31/35, 88\%). Four patients had moderately severe bleeding that required blood transfusion of $\leq 4$ units of packed red blood cells. All patients with post-ERCP bleeding were successfully treated by endoscopic methods $(n=25)$ or were self-limited $(n=10)$. There was no mortality.

\section{Risk factor of post-ERCP bleeding}

In the univariate analysis, post-ERCP bleeding was associated with age, pull-type sphincterotomy, aspirin, single APA, and multiple APAs. In contrast, balloon dilation of the biliary orifice and needle-knife sphincterotomy were inversely associated with post-ERCP bleeding (Table 3). There was no greater risk for bleeding for patients who had their drug(s) held for 0 to 2 days compared to those who had them held for 3 to 6 days.

In the multivariate analysis, pull-type sphincterotomy (odds ratio [OR], 7.829; 95\% confidence interval [CI], 1.411 to 43.453 ; $\mathrm{p}=0.019$ ) and country (Korea: OR, 0.124; 95\% CI, 0.042 to 0.361; $\mathrm{p}<0.001)$ were associated with post-ERCP bleeding (Table 4).
Table 4. Multivariate Analysis of the Risk Factors for Post-ERCP Bleeding

\begin{tabular}{|c|c|c|}
\hline Factor & OR (95\% CI) & $p$-value \\
\hline Age & $0.999(0.974-1.026)$ & 0.957 \\
\hline \multicolumn{3}{|l|}{ Sex } \\
\hline Male & 1 & \\
\hline Female & $1.029(0.486-2.177)$ & 0.941 \\
\hline \multicolumn{3}{|l|}{ Country } \\
\hline USA & 1 & \\
\hline Korea & $0.124(0.042-0.361)$ & $<0.001$ \\
\hline \multicolumn{3}{|l|}{ Intervention } \\
\hline Pull-type sphincterotomy & $7.829(1.411-43.453)$ & 0.019 \\
\hline Needle-knife sphincterotomy & $0.412(0.093-1.831)$ & 0.244 \\
\hline Balloon dilation of the biliary orifice & $0.433(0.142-1.320)$ & 0.141 \\
\hline \multicolumn{3}{|l|}{ Drug } \\
\hline None & 1 & \\
\hline ASA alone & $0.457(0.143-1.456)$ & 0.185 \\
\hline Single APA other than ASA & $1.789(0.382-8.377)$ & 0.460 \\
\hline Multiple APA & $1.080(0.254-4.595)$ & 0.917 \\
\hline
\end{tabular}

ERCP, endoscopic retrograde cholangiopancreatography; OR, odds ratio; CI, confidence interval; ASA, acetylsalicylic acid; APA, antiplatelet agent.

\section{DISCUSSION}

This analysis of prospectively collected databases on postERCP bleeding showed that the frequency of post-ERCP bleeding increased in patients who had taken APAs within 6 days of ERCP. However, in the multivariate analysis, the type of APA, combination of APAs, and number of days the APAs were held were not significantly associated with post-ERCP bleeding. Only the performance of pull-type sphincterotomy was strongly associated with post-ERCP bleeding in the multivariate analysis.

The overall frequency of post-ERCP bleeding in the current study was $1.4 \%$. The previously reported frequencies ranged from $0.3 \% \%^{3}$ to $1.4 \% \%^{9,10}$ and its risk was increased with endoscopic sphincterotomy in three large-scaled studies. The frequency of post-ERCP bleeding among the patients on any APA within 6 days was 5.4\% in this study. In a retrospective study, postERCP bleeding occurred in 9.8\% of patients who continued taking APA within a week prior to endoscopic sphincterotomy, and there was no significant difference in post-ERCP bleeding when compared in terms of the APA used. ${ }^{11}$

The predictors for post-ERCP bleeding determined in previously published prospective studies were coagulopathy before the procedure, anticoagulation within three days after procedure, cholangitis before the procedure, low case volume, and precut sphincterotomy. ${ }^{1,12,13}$

In the multivariate analysis of the current study, pull-type sphincterotomy was an independent predictor for post-ERCP 
bleeding. However, the APA use prior to ERCP was not found to be predictive of bleeding. This finding may result from the small number of patients in the single and multiple APA groups. Therefore, until a large study adequately powered to detect differences in bleeding, caution is recommended when considering invasive procedures during ERCP in patients on APAs.

The country Korea was inversely associated with post-ERCP bleeding. In the patients enrolled in the study from Korea, balloon dilation of the biliary orifice consisted of 60\% of interventions performed (716 of 1,184 interventions, data not shown), compared to $7.4 \%$ in the United States. In addition, even though balloon dilation of the biliary orifice was not inversely associated with post-ERCP bleeding in the multivariate analysis, balloon dilation of the biliary orifice showed an OR of 0.119 in the univariate analysis. Use of balloon dilation instead of pull-type sphincterotomy may reduce the risk of post-ERCP bleeding, but clearly increases the risk of post-ERCP pancreatitis. ${ }^{14}$

There are several limitations in this study. First, as this study is a retrospective analysis of a prospectively collected database, only limited numbers of predictors for post-ERCP bleeding were included. Secondly, the number of patients on APAs was relatively small even though a large number of patients were included from three institutions over 4-year period. It is likely that many patients undergoing elective ERCPs held their APAs for at least 7 days because of the concern of post-ERCP bleeding. Finally, while the bleeding rate in this study is similar to that reported by others, ${ }^{9,10}$ the relatively low rate may explain our inability to identify other potential risk factors for bleeding including APAs. Thus, further study of larger populations of patients on APAs will be required to make a more definitive conclusion regarding the risk of APAs in ERCP.

In conclusion, the frequency of post-ERCP bleeding was higher in patients who had taken APAs within 6 days prior to ERCP but was not found to be a risk factor in the multivariate analysis. Pull-type sphincterotomy was an independent risk factor associated with post-ERCP bleeding. Even though APA use was not associated with post-ERCP bleeding in our study, until a large study adequately powered to detect differences in bleeding, caution is recommended when considering invasive procedures during ERCP in patients on APAs.

\section{CONFLICTS OF INTEREST}

No potential conflict of interest relevant to this article was reported.

\section{ACKNOWLEDGEMENTS}

Author contributions: H.C.O., study design, analysis, drafting;
J.J.E., data collection, drafting; I.I.E.H., J.W., E.L.F., L.M., J.S.C., data collection; G.A.L., data collection, supervision; H.K., statistical analysis; S.S., study design, supervision, drafting.

\section{REFERENCES}

1. Freeman ML, Nelson DB, Sherman S, et al. Complications of endoscopic biliary sphincterotomy. N Engl J Med 1996;335:909-918.

2. Andriulli A, Loperfido S, Napolitano G, et al. Incidence rates of post-ERCP complications: a systematic survey of prospective studies. Am J Gastroenterol 2007;102:1781-1788.

3. Cotton PB, Garrow DA, Gallagher J, Romagnuolo J. Risk factors for complications after ERCP: a multivariate analysis of 11,497 procedures over 12 years. Gastrointest Endosc 2009;70:80-88.

4. Freeman ML. Adverse outcomes of ERCP. Gastrointest Endosc 2002;56(6 Suppl):S273-S282.

5. ASGE Standards of Practice Committee, Acosta RD, Abraham NS, et al. The management of antithrombotic agents for patients undergoing GI endoscopy. Gastrointest Endosc 2016;83:3-16.

6. Veitch AM, Vanbiervliet G, Gershlick AH, et al. Endoscopy in patients on antiplatelet or anticoagulant therapy, including direct oral anticoagulants: British Society of Gastroenterology (BSG) and European Society of Gastrointestinal Endoscopy (ESGE) guidelines. Gut 2016;65:374-389.

7. Cotton PB, Lehman G, Vennes J, et al. Endoscopic sphincterotomy complications and their management: an attempt at consensus. Gastrointest Endosc 1991;37:383-393.

8. Ferreira LE, Baron TH. Post-sphincterotomy bleeding: who, what, when, and how. Am J Gastroenterol 2007;102:2850-2858.

9. Williams EJ, Taylor S, Fairclough P, et al. Risk factors for complication following ERCP: results of a large-scale, prospective multicenter study. Endoscopy 2007;39:793-801.

10. Wang P, Li ZS, Liu F, et al. Risk factors for ERCP-related complications: a prospective multicenter study. Am J Gastroenterol 2009;104:31-40.

11. Lee MG, Kim J, Lee SH, et al. Effect of sustained use of platelet aggregation inhibitors on post-endoscopic sphincterotomy bleeding. Dig Endosc 2014;26:737-744.

12. Loperfido S, Angelini G, Benedetti G, et al. Major early complications from diagnostic and therapeutic ERCP: a prospective multicenter study. Gastrointest Endosc 1998;48:1-10.

13. Masci E, Toti G, Mariani A, et al. Complications of diagnostic and therapeutic ERCP: a prospective multicenter study. Am J Gastroenterol 2001;96:417-423.

14. Disario JA, Freeman ML, Bjorkman DJ, et al. Endoscopic balloon dilation compared with sphincterotomy for extraction of bile duct stones. Gastroenterology 2004;127:1291-1299. 